\title{
ANÁLISE DA COMUNIDADE FITOPLANCTÔNICA NA REPRESA DE FURNAS EM BARRANCO ALTO - MG
}

\author{
Lúcia Wanessa Bressane ${ }^{1}$
}

Renata Martins dos Santos ${ }^{2}$

Roberta Brandão Mendes ${ }^{3}$

RESUMO: O fitoplâncton é formado por organismos microscópicos fotossintetizantes muito importantes para o meio ambiente aquático, devido a sua importância na produção primária e como um indicador eficiente das alterações do ecossistema. O presente estudo teve como objetivo uma análise qualitativa e quantitativa da comunidade fitoplanctônica na Represa de Furnas, no distrito de Barranco Alto, Minas Gerais onde foram realizadas coletas em três pontos no mês de abril de 2013. Foram analisadas variáveis físicas e químicas da água, que mostraram uma grande concentração de nitrogênio total, fósforo total, nitrato e amônia no ponto 1. $\mathrm{Na}$ análise da comunidade fitoplanctônica, foram identificadas oito classes, totalizando 30 táxons. Houve uma grande variedade de espécies da classe Cyanophyceae, seguida pela Bacillariophyceae. A maior riqueza de espécies foi registrada no P2 (21 espécies) e a menor no P1 (13 espécies). A espécie Cylindrospermopsis raciborskii, dentre as cianofíceas, Aulacoseira granulata dentre as diatomáceas e Ceratium furcoides, dentre os dinoflagelados, foram registradas em

\footnotetext{
${ }^{1}$ Graduanda da Universidade do Vale do Sapucaí. E-mail: lucia_wanessa@hotmail.com

${ }^{2}$ Pós graduanda em Ecologia e Recursos Naturais na Universidade Federal de São Carlos. E-mail: renata_cordis@yahoo.com.br

${ }^{3}$ Graduanda da Universidade do Vale do Sapucaí. E-mail: ro atry@hotmail.com
} 
elevadas densidades. Essa última espécie, invasora no local, foi a dominante em todos os pontos amostrados. Todos os dados apontam para uma possível poluição causada por esgoto doméstico e adubos de plantações locais despejados na represa no P1. Observase a necessidade de medidas preventivas no local para que não ocorra um progresso da eutrofização no reservatório de Furnas.

Palavras-chave: Fitoplâncton. Reservatório de Furnas. Eutrofização.

\section{INTRODUÇÃO}

Em suspensão nos ambientes aquáticos encontram-se um conjunto de organismos microscópicos fotossintetizantes denominado fitoplâncton, que é sujeito ao movimento passivo provocado por ventos e correntes (REYNOLDS, 1984). Ele possui uma grande importância na diversidade aquática, com várias espécies em todo o mundo; sendo sua estimativa maior calculada em 40.000 espécies (WILSON, 1992) e a menor em 26.900 espécies (HAMMOND, 1992).

Segundo resultados de pesquisas recentes foram confirmados a ocorrência de 3.497 espécies (1.988 epicontinentais e 1.541 marinhas) reunidas em 829 gêneros e 17 classes de algas. O Brasil possui 2 gêneros e 52 espécies endêmicas referentes ao valor total (MENEZES \& BICUDO, 2010).

O estudo da comunidade fitoplanctônica, é relevante por sua importância na produção primária do ambiente aquático, como indicador eficiente das alterações decorrentes no ecossistema e, além disso, o seu curto ciclo de vida permite a compreensão de importantes processos, tornando-a um modelo para o entendimento de outras comunidades e dos ecossistemas em geral (BOZELLI \& HUSZAR, 2003).

A variedade de algas dos ecossistemas aquáticos continentais possui alta proporção na biodiversidade total destes sistemas. Qualquer mudança referente em termos de espécie e/ou biomassa pode afetar a fotossíntese, a utilização de nutrientes, as taxas de predação e, consequentemente, interferir nos níveis mais elevados da cadeia alimentar (WETZEL \& LIKENS, 1979). 
Fórum Ambiental

da Alta Paulista
ISSN 1980-0827

Volume 9, Número 2, 2013

Bacias Hidrográficas, Planejamento e Gestão dos Recursos Hídricos



ANAP

Qualquer mudança qualitativa e ou quantitativa na comunidade fitoplanctônica podem ter importante significado para vários componentes do ecossistema e, até mesmo, inviabilizar o uso da água (TUCCI, 2002).

Dessa forma, o presente estudo tem como objetivo principal realizar uma análise qualitativa e quantitativa da comunidade fitoplanctônica no reservatório de Furnas no distrito de Barranco Alto, Minas Gerais.

\section{METODOLOGIA}

\section{1 Área de estudo}

O reservatório de Furnas está localizado na Bacia do Rio Grande, no Sul de Minas Gerais. Possuindo uma extensão máxima de 220 km, com área inundada de 1.440 km², volume total de 22,95 bilhões de $\mathrm{m}^{3}$, profundidade máxima (na barragem) de $100 \mathrm{~m}$ e profundidade média de 15,5 m (SÁ et. al. 1996). Composto por dois grandes braços, Rio Grande e Rio Sapucaí, sua barragem situa-se no curso médio do Rio Grande, no trecho denominado "Corredeiras de Furnas", entre os municípios de São José da Barra e São João Batista do Glória.



Figura 1. Mapa da localização do reservatório de Furnas, Minas Gerais, Brasil. (Modificado de SILVA et al., 2012) 


\subsection{Amostragem}

A pesquisa foi realizada no reservatório de Furnas no distrito de Barranco Alto, no município de Alfenas (MG) em três pontos de amostragem: P1 (21010'98"S e

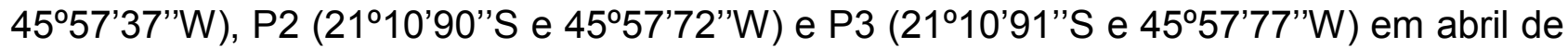
2013.

\subsection{Variáveis físicas e químicas da água}

Em cada ponto de amostragem, por meio do multisensor Horiba U10 foram determinadas in situ as variáveis limnológicas (condutividade, oxigênio dissolvido, pH e temperatura) e também foram coletadas amostras de água para a determinação das concentrações de nutrientes, nitrogênio e fósforo total (VALEDRRAMA, 1981); fosfato inorgânico e fosfato total dissolvido (STRICKLAND \& PARSONS,1960); nitrito e silicato (GOLTERMAN et al.,1978); nitrato (MACKERETH et al.,1978) e amônio (KOROLEFF, 1976).

\subsection{Comunidade fitoplanctônica}

As amostras de fitoplâncton foram coletadas com o auxílio de rede de plâncton com $20 \mu \mathrm{m}$ de abertura de malha e de $25 \mathrm{~cm}$ de diâmetro, sendo filtrados 100 litros de água. As amostras foram transferidas para frascos de polietileno, preservadas com formol a $4 \%$. A identificação dos táxons foi feita até o nível genérico e, quando possível, até o nível específico. O sistema de classificação utilizado foi o Hoeck et al. (1995) e a identificação dos taxa foi feita utilizando-se as seguintes bibliografias: para a classe Cyanophyceae Anagnostidis \& Komárek (1989) e Komárek \& Anagnostidis (1999, 2005); e para as demais classes: Chlorophyceae, Zygnematophyceae, Cryptophyceae, Bacillariophyceae, Dinophyceae - Bicudo \& Menezes (2005).

A contagem foi realizada em câmara de Sedgwick-Rafter, contando-se um $\mathrm{mL}$ em microscópio óptico com aumento de 100X.

\subsection{Análise dos dados}




\section{T Fon

Os índices da riqueza taxonômica; diversidade de Shannon-Wiener $\left(\mathrm{H}^{\prime}\right)$ (SHANNON \& WEAVER, 1949) e dominância entre os pontos amostrados foram calculados utilizando o programa Past-Paleontological Statistics Software (HAMMER et al.,2001).

O índice de similaridade de Jaccard baseado na presença ausência das espécies fitoplanctônicas entre os pontos também foi calculado utilizando o programa PastPaleontological Statistics Software.

A frequência de ocorrência das espécies foi calculada segundo Dajoz (1983), levando-se em consideração o número de amostras onde o organismo ocorreu, em relação ao número total das amostras coletadas (em porcentagem), de acordo com a fórmula a seguir:

$$
\mathrm{Fo}=\mathrm{Ta} \times 100 / \mathrm{TA}
$$

Onde:

$\mathrm{Ta}=\mathrm{n}^{\circ}$ de amostragem em que o táxon ocorreu

$\mathrm{TA}=\mathrm{n}^{\circ}$ total de amostragem relacionada

Foram consideradas as seguintes categorias:

Muito Frequêntes: Fo > 70;

Frequêntes: $40<$ Fo $\leq 70$;

Pouco Frequêntes: $20<$ Fo $\leq 40$;

Esporádicas: Fo $\leq 20$.

Correlações de Pearson entre os fatores abióticos e bióticos (densidade das classes fitoplanctônicas) foram calculadas, envolvendo os dados de todos os pontos de coleta. Essa análise foi realizada utilizando o programa Past-Paleontological Statistics Software (HAMMER et al., 2001).

\section{RESULTADOS}

\subsection{Variáveis físicas e químicas da água}




\section{Then Fórum Ambiental \\ da Alta Paulista

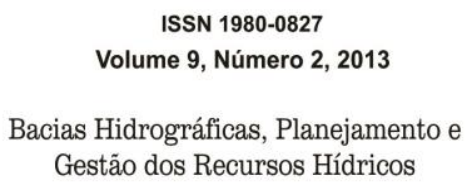

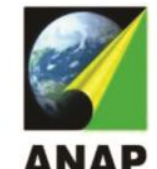

Entre os pontos de amostragem não foi registrado diferenças significativas das variáveis físicas e químicas avaliadas ( $p>0,05)$ (Tabela 1). No entanto, verifica-se que no ponto $\mathrm{P} 1$ foram registrados os maiores valores de fósforo total $\left(31,4 \mu \mathrm{g} \mathrm{L}^{-1}\right)$, nitrogênio total $\left(122,96 \mu \mathrm{g} \mathrm{L}^{-1}\right)$, nitrato $\left(50,93 \mu \mathrm{g} \mathrm{L}^{-1}\right)$ e de amônia $\left(67,18 \mu \mathrm{g} \mathrm{L}^{-1}\right)$.

Tabela 1. Variáveis físicas e químicas da água registradas nos pontos amostrais no reservatório de Furnas em abril de 2013. (DP = desvio padrão).

\begin{tabular}{|c|c|c|c|c|c|}
\hline & P1 & $\mathbf{P 2}$ & P3 & Média & DP \\
\hline pH & 6,9 & 6,79 & 7,42 & 7,0 & 0,3 \\
\hline Condutividade elétrica $\left(\mu \mathrm{S} \mathrm{cm}^{-1}\right)$ & 34 & 32 & 33 & 33,0 & 1,0 \\
\hline Temperatura da água $\left({ }^{\circ} \mathrm{C}\right)$ & 24,07 & 25,43 & 25,9 & 25,1 & 1,0 \\
\hline Oxigênio dissolvido $\left(\mathrm{mg} \mathrm{L}^{-1}\right)$ & 7,91 & 7,52 & 7,48 & 7,6 & 0,2 \\
\hline Fósforo Total $\left(\mu \mathrm{g} \mathrm{L}^{-1}\right)$ & 31,4 & 31,08 & 29,77 & 30,8 & 0,9 \\
\hline Fosfato Total Dissolvido ( $\mu \mathrm{g} \mathrm{L-1})$ & 9,73 & 12,79 & 12,48 & 11,7 & 1,7 \\
\hline Fosfato Orgânico $\left(\mu \mathrm{g} \mathrm{L}^{-1}\right)$ & 3,23 & 4,23 & 4,22 & 3,9 & 0,6 \\
\hline Fosfato Inorgânico ( $\mu \mathrm{g} \mathrm{L-1)}$ & 6,51 & 8,56 & 8,27 & 7,8 & 1,1 \\
\hline Nitrogênio Total $\left(\mu \mathrm{g} \mathrm{L}^{-1}\right)$ & 122,96 & 89,69 & 64,91 & 92,5 & 29,1 \\
\hline Nitrito $\left(\mu \mathrm{g} \mathrm{L}^{-1}\right)$ & 0,13 & 0,3 & 0,07 & 0,2 & 0,1 \\
\hline Nitrato $\left(\mu \mathrm{g} \mathrm{L}^{-1}\right)$ & 50,93 & 23,54 & 15,99 & 30,2 & 18,4 \\
\hline Amônio $\left(\mu \mathrm{g} \mathrm{L}^{-1}\right)$ & 67,18 & 46,97 & 40,75 & 51,6 & 13,8 \\
\hline Silicato $\left(\mu \mathrm{g} \mathrm{L}^{-1}\right)$ & 4,34 & 7,43 & 5,49 & 5,8 & 1,6 \\
\hline
\end{tabular}

\subsection{Comunidade fitoplanctônica}

No total foram identificadas oito classes fitoplanctônicas contendo 30 táxons no total. A família com maior número de representantes foi a Cyanophyceae com 12 taxons, seguida por Bacillariophyceae (sete táxons) e Chlorophyceae (quatro), juntas estas representaram mais de $76 \%$ da riqueza total (Tabela 2). Entre as demais Zygnematophycae registrou três táxons e Oedogoniophyceae, Cryptophyceae, Dinophyceae e Euglenophyceae apenas um táxon cada uma.

As espécies mais freqüentes, ou seja, que ocorreram em todos os pontos amostrais foi Cylindrospermopsis raciborskii entre as cianofíceas, Aulacoseira granulata entre as diatomáceas e Ceratium furcoides entre os dinoflagelados. As demais espécies foram classificadas como freqüentes ou pouco freqüentes. 
A maior riqueza de espécies foi registrada no ponto P2 (21 espécies) e a menor no ponto P1 (13 espécies). De uma forma geral a contribuição na riqueza de espécies entre as classes seguiu o padrão do sistema como um todo, com maior diversidade de Cyanophyceae seguida por Bacillariophyceae.

Tabela 2. Lista das espécies da comunidade fitoplanctônica registradas nos pontos amostrais no reservatório de Furnas em abril de 2013. (FO = frequência de ocorrência).

\begin{tabular}{|c|c|c|c|c|c|}
\hline Taxa & P1 & P2 & P3 & $\%$ & FO \\
\hline \multicolumn{6}{|l|}{ Cyanophyta } \\
\hline \multicolumn{6}{|l|}{ Cyanophyceae } \\
\hline Cylindrospermopsis raciborskii & $\mathrm{x}$ & $x$ & $x$ & 100,0 & Muito frequente \\
\hline Dolichospermum spiralis & $\mathrm{X}$ & & & 33,3 & Pouco frequente \\
\hline Geitlerinema & & & $x$ & 33,3 & Pouco frequente \\
\hline Leptolyngbya perelegans & $\mathrm{X}$ & $\mathrm{X}$ & & 66,7 & Frequente \\
\hline Microcystis aeruginosa & & $x$ & $x$ & 66,7 & Frequente \\
\hline Phormidium sp. & $\mathrm{x}$ & & $x$ & 66,7 & Frequente \\
\hline Planktolyngbya limnetica & $\mathrm{x}$ & $x$ & & 66,7 & Frequente \\
\hline Pseudanabaena mucicola & & $\mathrm{X}$ & & 33,3 & Pouco frequente \\
\hline Pseudanabaena sp. & & & $x$ & 33,3 & Pouco frequente \\
\hline Pseudolyngbya sp. & $\mathrm{X}$ & & & 33,3 & Pouco frequente \\
\hline Raphidiopsis mediterranea & & $x$ & & 33,3 & Pouco frequente \\
\hline Snowella lacustres & & $\mathrm{X}$ & $x$ & 66,7 & Frequente \\
\hline \multicolumn{6}{|l|}{ Chlorophyta } \\
\hline \multicolumn{6}{|l|}{ Chlorophyceae } \\
\hline Ankistrodesmus fusiformes & & & $x$ & 33,3 & Pouco frequente \\
\hline Eutetramorus fottii & & $\mathrm{X}$ & $x$ & 66,7 & Frequente \\
\hline Radiococcus sp. & & $x$ & $x$ & 66,7 & Frequente \\
\hline Tetraedron minimum & & $\mathrm{X}$ & & 33,3 & Pouco frequente \\
\hline \multicolumn{6}{|l|}{ Zygnematophyceae } \\
\hline Closterium parvulum & $\mathrm{X}$ & $\mathrm{X}$ & & 66,7 & Frequente \\
\hline Staurastrum leptocladum & $x$ & & & 33,3 & Pouco frequente \\
\hline Staurastrum sp. & & $\mathrm{X}$ & & 33,3 & Pouco frequente \\
\hline \multicolumn{6}{|l|}{ Oedogoniophyceae } \\
\hline Oedogonium sp. & & $\mathrm{X}$ & $x$ & 66,7 & Frequente \\
\hline \multicolumn{6}{|l|}{ Heterokontophyta } \\
\hline \multicolumn{6}{|l|}{ Bacillariophyceae } \\
\hline Aulacoseira granulata & $x$ & $\mathrm{X}$ & $x$ & 100,0 & Muito frequente \\
\hline Cyclotela sp. & $x$ & & & 33,3 & Pouco frequente \\
\hline
\end{tabular}




\begin{tabular}{|c|c|c|c|c|c|}
\hline Fragilaria sp. & & $\mathrm{X}$ & $X$ & 66,7 & Frequente \\
\hline Navicula sp. & & $\mathrm{X}$ & $X$ & 66,7 & Frequente \\
\hline Pinnularia sp. & $x$ & $x$ & $x$ & 100,0 & Muito frequente \\
\hline Surirella sp. & & $x$ & $x$ & 66,7 & Frequente \\
\hline Tabellaria sp. & $x$ & $x$ & $x$ & 100,0 & Muito frequente \\
\hline \multicolumn{6}{|l|}{ Cryptophyta } \\
\hline \multicolumn{6}{|l|}{ Cryptophyceae } \\
\hline Cryptomonas sp. & & & $\mathrm{X}$ & 33,3 & Pouco frequente \\
\hline \multicolumn{6}{|l|}{ Dinophyta } \\
\hline \multicolumn{6}{|l|}{ Dinophyceae } \\
\hline Ceratium furcoides & $\mathrm{X}$ & $x$ & $X$ & 100,0 & Muito frequente \\
\hline \multicolumn{6}{|l|}{ Euglenophyta } \\
\hline \multicolumn{6}{|l|}{ Euglenophyceae } \\
\hline Trachelomonas volvocina & & $\mathrm{X}$ & & 33,3 & Pouco frequente \\
\hline Total & 13 & 21 & 18 & & \\
\hline
\end{tabular}

A espécie Ceratium furcoides foi registra em altas densidades em todos os pontos amostrados (Tabela 3). Essa espécie no ponto P3 chegou a representar $85 \%$ da abundância total $\left(38,28\right.$ org $\left.\mathrm{L}^{-1}\right)$. Cylindrospermopsis raciborskii também registrou valores expressivos de densidade em todos os pontos.

Tabela 3. Densidade (org $\mathrm{L}^{-1}$ ) das espécies da comunidade fitoplanctônica registradas nos pontos amostrais no reservatório de Furnas em abril de 2013.

\begin{tabular}{|c|c|c|c|}
\hline Taxa & P1 & $\mathbf{P 2}$ & P3 \\
\hline \multicolumn{4}{|l|}{ Cyanophyta } \\
\hline \multicolumn{4}{|l|}{ Cyanophyceae } \\
\hline Cylindrospermopsis raciborskii & 1,74 & 2,1 & 2,64 \\
\hline Dolichospermum spiralis & 0,24 & & \\
\hline Microcystis aeruginosa & & 0,06 & 0,18 \\
\hline Phormidium sp. & 0,12 & & \\
\hline Pseudanabaena sp. & & & 0,84 \\
\hline Raphidiopsis mediterrânea & & 0,06 & \\
\hline \multicolumn{4}{|l|}{ Chlorophyta } \\
\hline \multicolumn{4}{|l|}{ Chlorophyceae } \\
\hline Eutetramorus fottii & & 0,18 & \\
\hline Radiococcus sp. & & 0,06 & \\
\hline \multicolumn{4}{|l|}{ Zygnematophyceae } \\
\hline Closterium parvulum & 0,06 & 0,06 & \\
\hline
\end{tabular}




\begin{tabular}{|c|c|c|c|}
\hline Staurastrum leptocladum & 0,06 & 0,06 & \\
\hline \multicolumn{4}{|l|}{ Oedogoniophyceae } \\
\hline Oedogonium sp. & & 0,48 & 0,06 \\
\hline \multicolumn{4}{|l|}{ Heterokontophyta } \\
\hline \multicolumn{4}{|l|}{ Bacillariophyceae } \\
\hline Aulacoseira granulata & 0,15 & 0,3 & 0,3 \\
\hline Cyclotela sp. & 0,06 & & \\
\hline Fragilaria sp. & & 0,72 & 0,18 \\
\hline Navicula sp. & & 2,1 & 0,72 \\
\hline Pinnularia sp. & 0,06 & 0,66 & 0,84 \\
\hline Surirella sp. & & 0,06 & \\
\hline Tabellaria sp. & 0,06 & 1,74 & 0,78 \\
\hline \multicolumn{4}{|l|}{ Dinophyta } \\
\hline \multicolumn{4}{|l|}{ Dinophyceae } \\
\hline Ceratium furcoides & 9,84 & 7,86 & 38,28 \\
\hline \multicolumn{4}{|l|}{ Euglenophyta } \\
\hline \multicolumn{4}{|l|}{ Euglenophyceae } \\
\hline Trachelomonas volvocina & & 0,06 & \\
\hline Total & 12,39 & 16,56 & 44,82 \\
\hline
\end{tabular}

Diferente da riqueza de espécies, a maior densidade das espécies do fitoplâncton foi registrada no ponto P3 $\left(44,82\right.$ org L $\left.\mathrm{L}^{-1}\right)$ e a menor no ponto P1 (12,39 org L $\left.\mathrm{L}^{-1}\right)$ (Figura 2).

A classe Dinophyceae foi mais abundante em todos os pontos amostrados. Com exceção do ponto $\mathrm{P} 2$, a segunda classe mais dominante em termos de densidade foi Cyanophyceae. Isso ocorreu, pois nesse ponto foi registrado elevada densidade de Navicula sp. $\left(2,1\right.$ org L $\left.{ }^{-1}\right)$. 


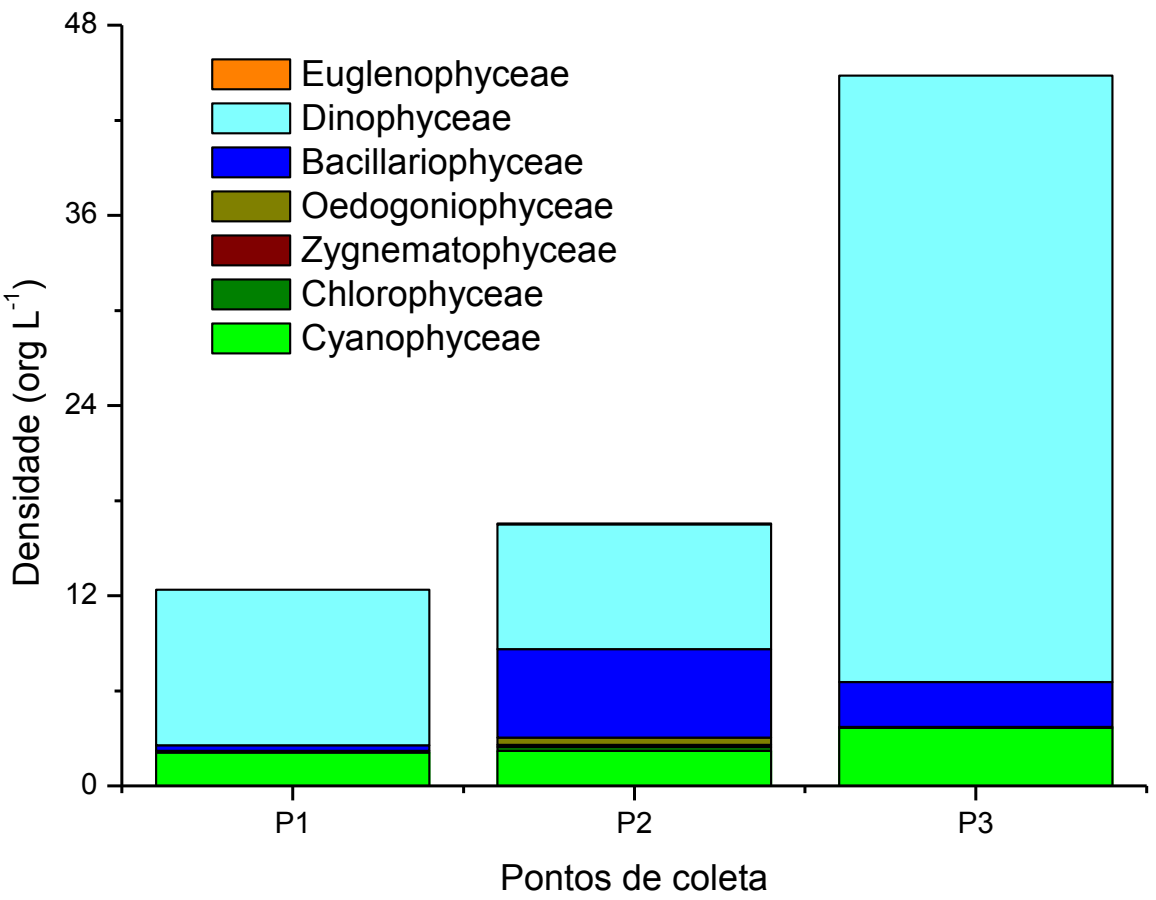

Figura 2. Densidade (org L ${ }^{-1}$ ) da comunidade fitoplanctônica registradas nos pontos de coleta no reservatório de Furnas em abril de 2013.

3.3 Análise dos dados

Ao analisar o dendrograma de similaridade de Jaccard (Figura 3), observa-se que os pontos 2 e 3 são mais similares entre si, apresentando cerca de $50 \%$ de semelhança. O ponto 1 foi dissimilar em relação aos demais. 


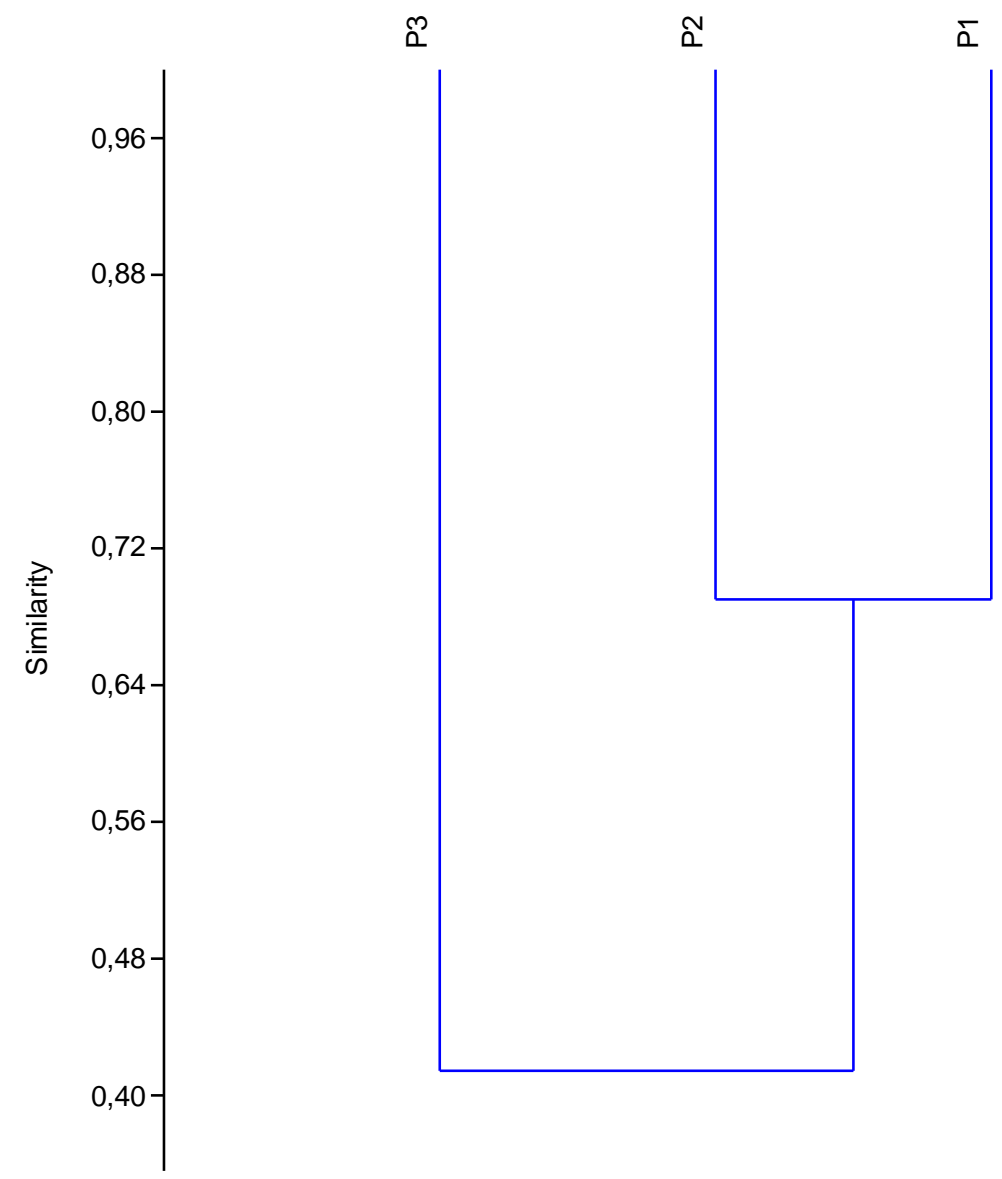

Figura 3. Dendrograma de similaridade de Jaccard baseado na presença/ausência das espécies da comunidade fitoplanctônica registradas nos pontos de coleta no reservatório de Furnas em abril de 2013. (coeficiente cofenético =1).

No geral, todos os pontos apresentaram baixos valores de diversidade (Figura 4). O ponto que apresentou maior valor do índice de diversidade foi P2 com 1,74 nats/ind., também o ponto com maior valor de equidade $(0,67)$ e o menor valor de dominância $(0,29)$. O oposto foi registrado no ponto P3, que foi o de menor diversidade, no entanto com uma alta dominância da espécie $C$. furcoides. 


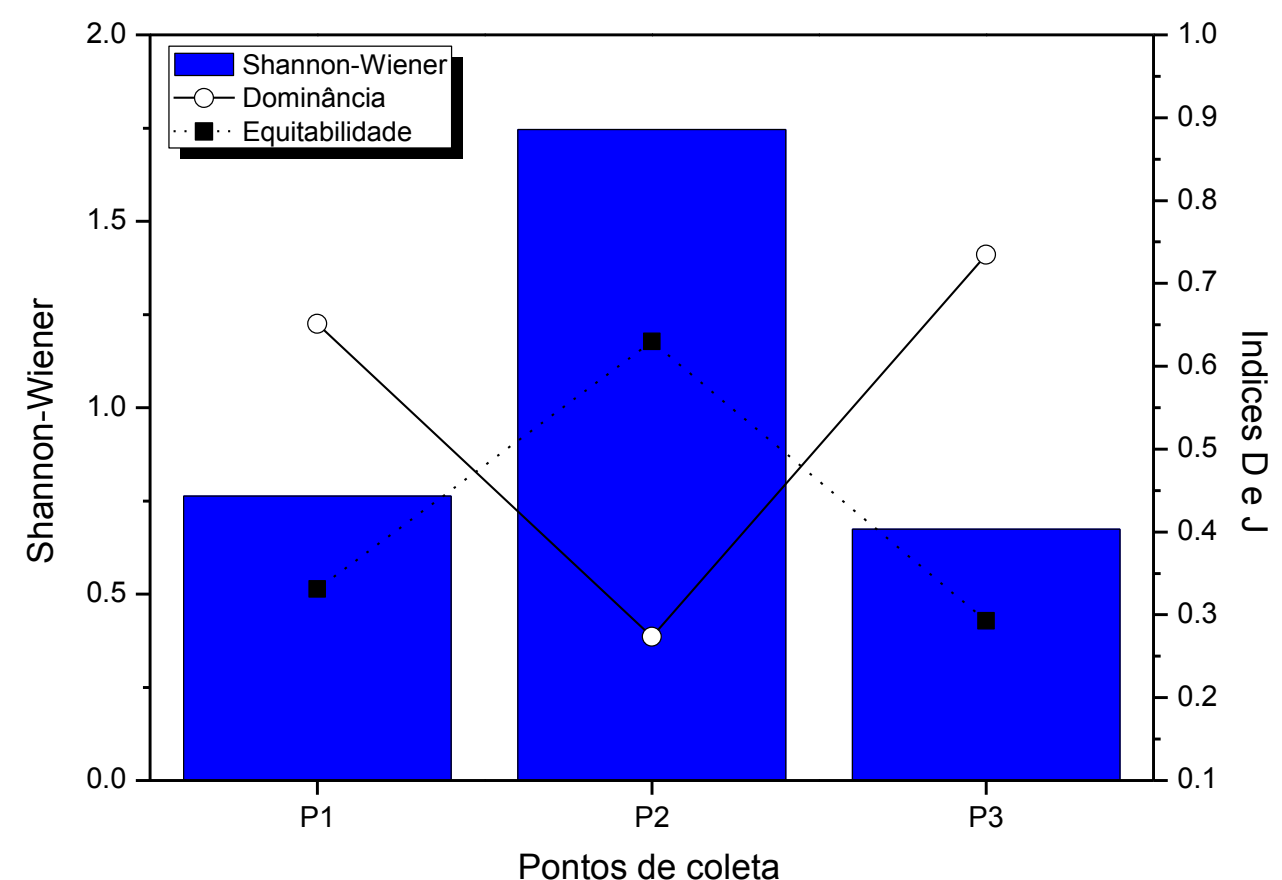

Figura 4. Índices de diversidade (Shannon-Wiener), dominância (D) e equitabilidade (J) da comunidade fitoplanctônica nos pontos de coleta no reservatório de Furnas em abril de 2013.

As densidades das classes fitoplanctônicas apresentaram altas correlações com as variáveis ambientais (Tabela 4). O silicato apresentou forte correlação positiva com a classe Bacillariophyceae $\left(r^{2}=0,9932\right)$, bem como com as densidades de Chlorophyceae, Oedogoniophyceae e Euglenophyceae. $\mathrm{O}$ pH também esteve positivamente correlacionado com as classes Cyanophyceae e Dinophyceae $\left(r^{2}=0,9729\right.$ e 0,9944 respectivamente) e negativamente correlacionado com Zygnematophyceae, Chlorophyecae e Euglenophyceae $\left(r^{2}=-0,9866\right.$ e -0,6348 respectivamente). Todas as classes, com exceção de Dinophyceae, apresentaram correlações negativas com a condutividade elétrica. Oedogoniophyceae e Baccilariophyceae foram as únicas que apresentaram forte correlação positiva com o fosfato total dissolvido $\left(r^{2}=0,6674\right.$ e 0,8956 respectivamente). Cyanophyceae foi a classe que apresentou maior correlação positiva com a temperatura da água $\left(r^{2}=0,7464\right)$. Os nutrientes nitrogenados dissolvidos apresentaram forte correlação com as densidades das classes fitoplanctônicas sendo que as maiores correlações foram registradas para Baccilariophyceae $\left(r^{2}=-0,7248\right.$ para 


\section{stom

nitrato), Chlorophyceae $\left(r^{2}=0,9679\right.$ para nitrito) e Cyanophyceae $\left(r^{2}=-0,7310\right.$ para amônio).

Tabela 4. Correlações de Pearson entre variáveis abióticas e bióticas (Cya = Cyanophyceae; Chl = Chlorophyceae; Zyg = Zygnemaphyceae; Oed = Oedogoniophyceae; $\mathrm{Bac}=$ Bacillariophyceae; Din = Dinophyceae; Eug = Euglenophyceae) nos três pontos de coleta no reservatório de Furnas em abril de 2013.

\begin{tabular}{|c|c|c|c|c|c|c|c|}
\hline & Cya & Chl & Zyg & Oed & Bac & Din & Eug \\
\hline Temperatura da água $\left({ }^{\circ} \mathrm{C}\right)$ & 0,7464 & 0,2703 & $-0,6986$ & 0,3790 & 0,6945 & 0,6558 & 0,2703 \\
\hline Oxigênio dissolvido (mg L' & $-0,6265$ & $-0,4253$ & 0,5711 & $-0,5263$ & $-0,8035$ & $-0,5224$ & $-0,4253$ \\
\hline Condutividade elétrica $\left(\mu \mathrm{S} \mathrm{cm}^{-1}\right)$ & $-0,0691$ & $-0,8660$ & $<0,000001$ & $-0,9177$ & $-0,9996$ & 0,0582 & $-0,8660$ \\
\hline pH & 0,9729 & $-0,6348$ & $-0,9866$ & $-0,5420$ & $-0,1927$ & 0,9944 & $-0,6348$ \\
\hline Fosfato Total Dissolvido ( $\mu \mathrm{g} \mathrm{L-1}$ ) & 0,4800 & 0,5776 & $-0,4182$ & 0,6674 & 0,8956 & 0,3646 & 0,5776 \\
\hline Nitrato $\left(\mu \mathrm{g} \mathrm{L}^{-1}\right)$ & $-0,7171$ & $-0,3115$ & 0,6672 & $-0,4185$ & $-0,7248$ & $-0,6227$ & $-0,3115$ \\
\hline Nitrito $\left(\mu \mathrm{g} \mathrm{L}^{-1}\right)$ & $-0,6508$ & 0,9679 & 0,7017 & 0,9326 & 0,7330 & $-0,7420$ & 0,9679 \\
\hline Amônio $\left(\mu \mathrm{g} \mathrm{L}^{-1}\right)$ & $-0,7310$ & $-0,2923$ & 0,6821 & $-0,4000$ & $-0,7107$ & $-0,6384$ & $-0,2923$ \\
\hline Silicato $\left(\mu \mathrm{g} \mathrm{L}^{-1}\right)$ & $-0,0773$ & 0,9298 & 0,1460 & 0,9659 & 0,9932 & $-0,2033$ & 0,9298 \\
\hline
\end{tabular}

\section{DISCUSSÃO}

Dentre os nutrientes necessários para o desenvolvimento do fitoplâncton, fósforo e nitrogênio são os principais, por serem considerados os que frequentemente limitam o desenvolvimento desta comunidade (REYNOLDS, 1997).

As variáveis físicas e químicas encontradas mostraram uma alta quantidade de fósforo, nitrogênio, nitrato e amônia. Esses valores podem estar relacionados com o nível de poluição da represa. Segundo Liliamtis (2007), uma poluição recente pode estar associada ao nitrogênio total ou na forma de amônia, enquanto que uma poluição de maior tempo associa-se ao nitrito e nitrato.

COQUEMALA (2005) cita que devido ao lançamento de esgoto não tratado, efluentes domésticos, lixiviação de solos degradados, fertilizantes e adubos ocorre um aumento de carga poluidora e consequentemente, grande concentração de matéria 
orgânica e nutrientes em reservatórios tropicais são registrados. Dessa forma, podemos destacar que devido a antropização do ambiente, há uma possível poluição por esgoto doméstico e adubos, levando em conta a proximidade dos pontos amostrais com plantações, sendo P1 (plantação de painço), P2 (plantação de banana) e P3 (plantação de eucalipto).

Em todos os pontos foram registrados baixos valores de diversidade, sendo o menor valor registrado no ponto P3. ODUM (1988) afirma que um número muito grande de organismos pertencentes a poucas espécies indica que o ambiente recebe carga poluente.

Algumas espécies de Cyanophyceae são indicadoras de eutrofização; onde predominam gêneros como Microcystis e Cylindrospermopsis (BROOKES, GANF e BURCH, 1998; REYNOLDS, 1998; SANT'ANNA e AZEVEDO, 2000; CHEN et al, 2003; TSUJIMURA e OKUBO, 2003; FERNANDES e LAGOS, 2003; FERNANDES, LAGOS e VENTURA, 2003; LATOUR et al., 2004). De acordo com os dados encontrados no reservatório de Furnas, eles reforçam uma possível poluição em fase inicial, levando em conta que se trata de um ambiente mesotrófico.

CETESB (2007) relata que o índice de estado trófico (IET) tem a finalidade de avaliar a qualidade da água, usando como referência os valores encontrados de fósforo total no ambiente (P). A classificação é feita de acordo com o Índice de Carlson, índice este que classifica ambientes mesotróficos com média de: $19<P \leq 52$. Levando em conta que a média de $\mathrm{P}$ encontrada nas coletas foi de 30,8 podemos considerar este ambiente como mesotrófico.

A análise quantitativa das amostras sugere uma grande densidade da espécie Ceratium furcoides em todos os pontos de coleta, sendo a espécie considerada uma invasora dominante do local. Nos resultados da pesquisa de NISHIMURA (2012), realizado nas represas de Billings e Guarapiranga, SP, observou-se a dominância pelo dinoflagelado invasor Ceratium furcoides na represa Billings. Outro estudo realizado no Reservatório de Furnas por Silva et al. (2012), relatou a invasão dessa mesma espécie, com maior densidade em ambiente mesotrófico, com um alto volume de esgoto doméstico sem tratamento sendo lançado diariamente. 
AMAZONA et al. (2012), ressalta que organismos pertencentes ao gênero Ceratium são encontrados em geral em águas eutrofizadas, vivendo em associação com organismos pertencentes a Cyanophyceae. O que comprova a grande variação de densidade da espécie Ceratium furcoides, que apresentou uma variação de 7,83 ind $\mathrm{mL}^{-1}$ no P2 a 38,28 ind $\mathrm{mL}^{-1}$ no P3. Neste último registrou-se também uma maior concentração de espécies pertencentes a classe Cyanophyceae representada principalmente por Cylindrospermopsis raciborskii.

Essa espécie foi mais abundante entre todas as Cyanophyceae, sendo classificada como muito frequente no presente trabalho. Um estudo realizado em três reservatórios em série no Ipiranga - SP, por LOPES (2007), demostrou a ocorrência de Cylindrospermopsis raciborskii como sendo abundante em todas as estações do ano. De acordo com PADISÁK (1997) que realizou um amplo estudo dessa espécie, sua abundância pode estar relacionada a sua alta afinidade pelo amônio e também a capacidade de armazenamento de fósforo.

\section{CONCLUSÃO}

As maiores concentrações dos nutrientes nitrogenados foram registradas no ponto $P 1$, onde se verificou o despejo de esgoto doméstico e utilização de adubos em plantações no entorno. Isso pode ter efeito direto no ambiente, afetando a qualidade da água e o prejudicando o desenvolvimento das espécies fitoplanctônicas.

Durante o período de estudo, verificou-se uma baixa diversidade da comunidade fitoplanctônica. Registrou-se a ocorrência e altas densidades da espécie invasora Ceratium furcoides, dominando em todos os pontos de coleta. Isso pode ser um indicador de mudanças na qualidade da água do reservatório de Furnas, uma vez que esta espécie só tinha ocorrência registrada no braço Sapucaí.

Através dos resultados encontrados, foi observado a necessidade de medidas preventivas na Represa de Furnas. Um possível reflorestamento das margens seria necessário para que o aporte de nutrientes provenientes das plantações da região seja reduzido e a implantação de sistemas de captação e tratamento de esgoto, para evitar o progresso da eutrofização no reservatório de Furnas. 


\section{AGRADECIMENTOS}

Os autores agradecem ao Departamento de Ecologia e Biologia Evolutiva da Universidade federal de São Carlos, em especial a prof ${ }^{a}$ Dra. Odete Rocha e ao Dr. José Valdecir de Lucca, pelas realização das análises dos nutrientes e emprestimo do material de campo.

\section{REFERÊNCIAS}

AMAZONAS et. al. Ocorrência de Ceratium furcoides (Levander) Langhans 1925 em Reservatórios do Estado de São Paulo, SP - Brasil. Campinas, 2012

BICUDO, C. E. M.; MENEZES, M. Introdução: As algas do Brasil. In: FORZZA, RC., org., et al. INSTITUTO DE PESQUISAS JARDIM BOTÂNICO DO RIO DE JANEIRO, 2010

BOZELLI, R. L.; HUSZAR, V. L. M. Comunidades fito e zooplanctônicas continentais em tempo de avaliação. Publicação da Sociedade Brasileira de Limnologia. Maio de 2003 p.6.

BROOKES, J. D.; GANF, G. G.; BURCH, M. D. Buoyancy regulation of Microcystis aeruginosa. E. Schweizerbart's Verlagsbuchhandlung. p. 1670-3, 1998.

CETESB Companhia de Tecnologia de Saneamento Ambiental. Relatório de qualidade das águas interiores do estado de São Paulo. São Paulo, 2007.

COQUEMALA, V. Variação Anual do Fitoplâncton no Reservatório de Passaúna, Paraná. Dissertação de Mestrado, Universidade Federal do Paraná, Curitiba, 92 p. 2005.

CHEN, Y.; QIN, B.; TEUBNER, K.; DOKULL, M. Long-term dynamics of phytoplankton assembleges: Microcystis - domination in Lake Taihu, a large shallow lake in China. Journal of Plankton Research, n. 1, v. 25, p. 445-453, 2003.

FERNANDES, L. F.; LAGOS, P. E. Florações de cianobactérias e eutrofização no reservatório do Iraí, Curitiba, PR. I: Sucessão sazonal do fitoplâncton. In: ANDREOLI, C. V. e CARNEIRO, C. (eds) Anais do IV Seminário do projeto interdisciplinar de pesquisa em eutrofização de águas de abastecimento público - tema: eutrofização de águas, 143p., 2003.

FERNANDES, L. F.; LAGOS, P. E.; VENTURA, C. Florações de cianobactérias e eutrofização no reservatório do Iraí, Curitiba, PR. II: Distribuição horizontal de algumas 


\section{S Fen Fórum Ambiental \\ da Alta Paulista

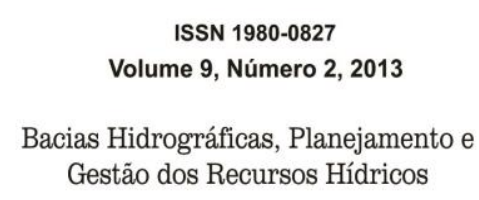

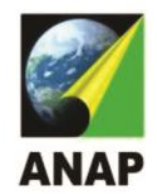

espécies. In: ANDREOLI, C. V. e CARNEIRO, C. (eds) Anais do IV Seminário do projeto interdisciplinar de pesquisa em eutrofização de águas de abastecimento público - tema: eutrofização de águas, 143p., 2003.

INAG, I.P. Manual para a avaliação da qualidade biológica da água. Protocolo de amostragem e análise para o Fitoplâncton. Ministério do Ambiente, do Ordenamento do Território e do Desenvolvimento Regional. Instituto da Água, I.P. 2009.

LATOUR, D.; SABIDO, O.; SALENÇON, M.; GIRAUDET, H. Dynamics and metabolic activity of benthic cyanobacterium Microcystis aeruginosa in the Grangent reservoir (France). Journal of Plankton research, n. 7, v. 26, p. 719-726, 2004.

LILIAMTIS T. B. Avaliação da adição de nitrato de amônio para redução de odor nos esgotos de Pereira Barretos - SP: Reflexos na qualidade da água do reservatório de Três Irmãos, após dez anos de aplicação. Tese de Doutorado, Faculdade de Saúde Pública da USP, São Paulo, 147 p. 2007.

LOPES, A. G. D. Estudo da Comunidade Fitoplanctônica como Bioindicador de Poluição em Três Reservatórios em Série do Parque Estadual das Fontes do Ipiranga (PEFI), Dissertação de Mestrado, Universidade de São Paulo, São Paulo, 137p. 2007.

NISHIMURA, P. Y. A Comunidade Fitoplanctônica nas Represas Billings e Guarapiranga ( Região Metropolitana de São Paulo), Tese de Doutorado, Universidade de São Paulo, São Paulo, 149p. 2012.

ODUM, E. P. Ecologia. Rio de Janeiro: Guanabara, 1988.

PADISÁK, J. Cylindrospermopsis raciborskii (Woloszynka) Seenaya et Subba Raju, na expanding, highly adaptive cyanobacterium: worldwide distribution and review of its ecology. Stuttgard. 563-593, 1997.

REYNOLDS, C.S. The Ecology of Freshwater phytophankton. Cambridge University Press, Oxford,1984. 384p.

REYNOLDS, C. S. Vegetation process in the pelagic: a model for ecosystem theory. In: KINNE, O. Excellence in ecology. Germany: Ecology Institute, 1997, 371p.

REYNOLDS, C. S. What factors influence the species composition of phytoplankton in lakes of different trophic status? Hydrobiologia, n. 370, p.11-26, 1998.

SÁ, W. P. J.; LIMA, M. N., EVANGELISTA, S. A. PERES, S.A., GOMES, A. P. Programa de monitoramento da Usina Hidrelétrica de Furnas. Estação de Hidrobiologia e Piscicultura de Furnas. 1996.

SANT'ANNA, C. L.; AZEVEDO, M. T. P. Contribution to the knowledge of potentially toxic Cyanobacteria from Brazil. Nova Hedwigia, n. 71, p. 359-385, 2000. 
SILVA, L. C.; LEONE, I. C.; SANTOS-WISNIEWSKI, M. J.; PERET, A. C.; ROCHA, O. Invasão do dinoflagelado Ceratium furcoides (Levander) Langhans $1925 \mathrm{em}$ um reservatório tropical e sua relação com as variáveis ambientais. Campinas, 2012.

TUCCI, A. Sucessão da Comunidade Fitoplanctônica de um Reservatório Urbano e Eutrófico, São Paulo, SP, Brasil. Tese de doutorado, Universidade Estadual Paulista Julio de Mesquita Filho, Rio Claro, 2002.

TSUJIMURA, S.; OKUBO, T. Development of Anabaena blooms in a small reservoir with dense sediment akinete population, with special reference to the temperature and irradiance. Journal of Plankton Research, n. 9, v. 25, p. 1059-1067, 2003.

WETZEL, R. G.; LIKENS, G. E. Limnological analysis. W.B. Saunders Co. Philadelphia . 357p. In: Wetzel, R.G. 2001. Limnology - Lake and River Ecossystems. 3ed. Academic. Press. USA,1979. 1006p.

WILSON, E. O. The Diversity of Life. Cambridge: Belknap Press, 1992.

Hammond, P. M. Species Inventory. In: Groombridge, B. (ed.). Global Biodiversity, Status of the Earth's Living Resources. London: Champman \& Hall, 1992. p. 17-39. 